\title{
Squeeze and Freeze, A Periodic Consecutive Oocyte Retrieval Method for Poor Ovarian Responders, Increases The Chance of Childbearing
}

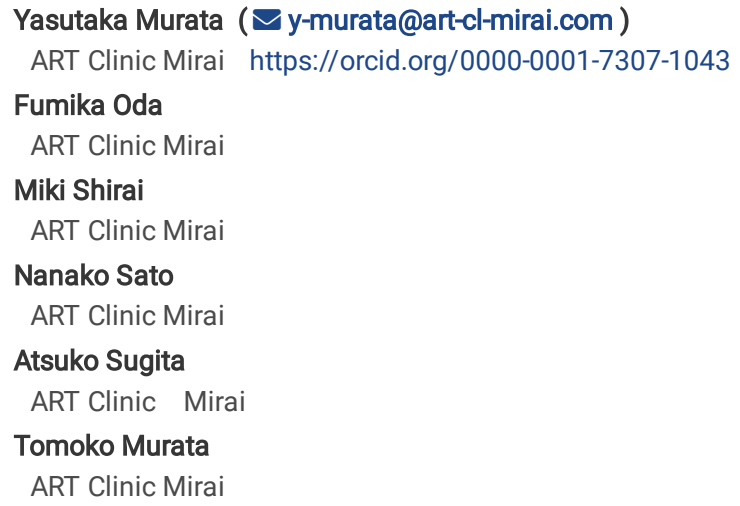

Research

Keywords: squeeze and freeze, consecutive oocyte retrievals, advanced maternal age, poor responder, luteal-phase stimulation

Posted Date: June 9th, 2021

DOI: https://doi.org/10.21203/rs.3.rs-560941/v1

License: (c) (i) This work is licensed under a Creative Commons Attribution 4.0 International License. Read Full License

Version of Record: A version of this preprint was published at Journal of Women's Health and Development on January 1 st, 2022. See the published version at https://doi.org/10.26502/fjwhd.2644-28840075. 


\section{Abstract \\ Purpose}

To assess if consecutive oocyte retrieval (OR) of more than three times could be an effective method to obtain competent embryos becoming babies.

\section{Methods}

To maximize the chances of pregnancy for refractory infertile women, we designed a new treatment course, "Squeeze and freeze." In this method, ovarian stimulation was initiated whenever the antral follicle was visible using ultrasound, regardless of the menstrual cycle stage for 3 months, aiming to retrieve all possible oocytes and collecting the maximum number of competent embryos for subsequent transfer. Eighty-eight patients underwent 101 treatment courses, in which retrievals at an average of 4.8 times were performed for 3 months. The clinical results and obstetric outcomes were retrospectively reviewed.

\section{Results}

The ratio of matured oocytes, 2PN embryos, available embryos, fair blastocysts per oocyte, and number of available embryos or fair blastocysts per retrieval did not differ between the two initial and later attempts. Similarly, no significant differences were observed in these parameters between the follicular- and luteal-phase retrievals. After subsequent embryo transfer, 62 patients achieved clinical pregnancy, 49 had an ongoing pregnancy, 47 delivered healthy babies, and 8 conceived a second baby.

\section{Conclusion}

Periodic consecutive OR is an efficient infertility treatment for refractory women. In this method, as many oocytes as possible are obtained from a patient in a limited period at the earliest stage of their life. More than three consecutive ORs, including luteal-phase ORs, could obtain competent embryos efficiently, increasing the chances of childbearing.

\section{Introduction}

Time is an important factor in infertility treatment, especially for women of advanced age or decreased ovarian reserve (DOR) [1-3]. In addition, as time progresses, the situation worsens as a result of maternal aging, further DOR, diminished number of oocytes retrieved, increased aneuploidy rate and miscarriage rate, and so on [4-7]. Among current in vitro fertilization (IVF) techniques, no therapy can restore the embryo's intrinsic competence, and the final infertility treatment outcomes depend on acquiring a potential embryo that will develop into a baby. To achieve this goal, the clinician should tailor the ovarian stimulation process to use the maximum ovarian reserve and retrieve the highest possible number of oocytes as early as possible in the patient's life.

Traditionally, ovarian stimulation was initiated in the early follicular phase because fresh transfer and a receptive endometrium are essential. However, with advanced vitrification techniques [8, 9], ovarian stimulation can be disconnected completely from the menstrual cycle, with no impact on the implantation rate, under the freeze-all strategy [10]. Recently, random-start ovarian stimulation has been proposed for cancer patients who desire emergency fertility preservation before beginning cancer treatment. In this process, ovarian stimulation begins randomly, at any phase in the menstrual cycle, and oocytes or embryos are preserved [11, 12]. Double ovarian stimulation (DuoStim) has also been proposed as method of harvesting more oocytes efficiently within the limited time available, providing double the opportunity for oocyte retrieval (OR) within a cycle [13-15]. Studies on these techniques have reported similar developmental potential of the oocytes retrieved from both follicular-phase stimulation (FPS) and luteal-phase stimulation (LPS), and subsequent frozen embryo transfer provides optimal pregnancy outcomes [16-19].

In this study, we report a combination technique using random-start and DuoStim. We further extend these ideas to establish a new treatment strategy called "SF." In this new scheme, consecutive ORs are planned within a specified time period (in this study, we selected 3 months) as far as the antral follicle is identified at any phase of the menstrual cycle. We squeeze out all possible oocytes and freeze all available embryos in the period. After all oocytes are retrieved, embryo transfers are planned. This unprecedented treatment course aims to maximize patient outcomes refractory to infertility treatment by obtaining the greatest number of eggs in a limited time at the earliest stage of their lives.

We termed this technique "squeeze and freeze" (SF course) and began using this method in our clinic for difficult outpatients in June 2017. Because of our institution's capacity, we invited a limited number of patients to participate, although many more hoped to adopt this course of treatment. Thus, this study represents a proof-of-concept investigation of the SF course of treatment.

\section{Materials And Methods}

\section{Study patients}

To maximize the chance of refractory infertile patients to achieve pregnancy, we designed the time-sensitive SF course to consist of 3 months of consecutive $\mathrm{OR}$. The aim of this treatment course is to acquire as many oocytes as possible in a limited period of time as early as possible in the patient's life. After fertilization and embryo culture, the acquired competent embryos, blastocysts in general, were vitrified for subsequent transfer. This process was performed quickly to minimize the amount of time lost as a result of futile embryo transfer or miscarriage. To participate in the SF course, patients had to meet at least 
one of the three following criteria: (1) maternal age $\geq 40$ years, (2) three or fewer oocytes retrieved after previous stimulation, and (3) reduced ovarian reserve (anti-Müllerian hormone $[\mathrm{AMH}]<1.0 \mathrm{ng} / \mathrm{mL}$ ). Between June 2017 and October 2018, we implemented 101 treatment courses and performed 485 OR cycles.

This was a retrospective cohort study conducted in a private fertility center in Japan. The study was approved by our clinic's Institutional Review Board and carried out in accordance with the Code of Ethics of the World Medical Association (Declaration of Helsinki) for experiments involving humans. All the candidates provided informed consent after receiving individual counseling about the time significance and merit and demerit of multiple consecutive stimulations. This study's main objective was to confirm the efficacy of the SF strategy for patients who are refractory to treatment and who fulfill the Bologna criteria [20]. We also examined whether more than three consecutive ORs and luteal-phase ORs could obtain competent embryos more efficiently, ultimately increasing the chance of childbearing.

\section{Ovarian stimulation protocol}

Ovarian stimulation was considered during any phase of the menstrual cycle, when one or more antral follicles was recognized by transvaginal ultrasound, to squeeze out all oocytes. In the first attempt, ovarian stimulation began on cycle day 2 to 4 , as is the normal convention, and a short, antagonist, or mild stimulation protocol was selected for each patient. Gonadotropin-releasing hormone (GnRH) agonist nasal spray (Buserelin acetate; Buserecur®, Fuji Pharm, Japan) and/or clomiphene citrate (CC; Clomid ${ }^{\circledR}$, Fuji Pharm, Japan)/aromatase inhibitor (Al; Femara ${ }^{\circ}$, Novartis, Basel, Switzerland) and/or gonadotropin injection were administered. Follicular monitoring was performed at the beginning, 5-7 days after the start and then as needed, continuing every $2-3$ days until the follicle reached approximately $18 \mathrm{~mm}$ in diameter. Serum follicle-stimulating hormone (FSH), luteinizing hormone (LH), estradiol, and progesterone levels were measured on ultrasound days using the analyzer Cobas e 411 plus (Roche, Basel, Switzerland), which uses electrochemiluminescence technology for immunoassay analysis. A GnRH antagonist was administered when the serum LH level was elevated or the leading follicle reached $16 \mathrm{~mm}$ in diameter. Final oocyte maturation was triggered by a GnRH agonist nasal spray and/or human chorionic gonadotropin (rec-hCG $250 \mu \mathrm{g}$; Ovidrel ${ }^{\circledR}$, Merck Biopharma, Darmstadt, Germany). Thirty-six hours after the trigger, ORs were performed via transvaginal ultrasound, in which all the follicles with a diameter greater than $10 \mathrm{~mm}$ were aspirated. The next trial began when the preceding trial resulted in a smaller number of retrieved oocytes (e.g., fewer than five), and at least one antral follicle was recognized by transvaginal ultrasound examination. In general, CC began the day after retrieval, when there were one or two visible antral follicles. When more than three antral follicles were visible, daily administration of 150 IU human menopausal gonadotropin (Ferring ${ }^{\circledR}$, Ferring Pharma, SaintPrex, Switzerland) was started following the same timing. After one week or longer, we attempted to retrieve the newly matured follicles in the luteal phase after administering the rec-hCG maturation trigger. Follicle monitoring was sometimes more difficult in the luteal phase because of the presence of corpora lutea and hormones; thus, we used serial ultrasound examinations and serum hormone measurements to help determine the timing. The same was true for the luteal-phase ORs, and careful examination helped distinguish the new follicles. When no follicle growth was detected after the seventh day, ovarian stimulation was stopped until the next menstruation. Thus, we attempted to repeat the OR as many times as possible for 3 months, regardless of the menstrual cycle.

\section{Embryo culture and assessment, and cryopreservation}

Sperm was collected after liquefaction and centrifugation and swim-up and then provided for conventional in vitro fertilization (IVF) or intracytoplasmic sperm injection (ICSI), depending on the semen condition. IVF was performed by inseminating each oocyte with $10^{5}$ motile spermatozoa/mL, and ICSI was performed after 2-4 $\mathrm{h}$ of incubation, after the cumulus and corona radiata cells were removed by hyaluronidase and pipetting. Embryos were cultured in the medium NX (Continuous Single Culture NX Complete Medium: IS Japan, Co. Ltd.) or S1 (SAGE 1-Step Medium: Origio Japan, Co. Ltd.) at 37 C in 6\% carbon dioxide and 5\% oxygen tension in a time-lapse incubator (Embryo Scope plus; Vitrolife, Co. Ltd., Göteborg, Sweden). Embryos that developed to the blastocyst stage were graded according to Gardner's criteria. A blastocyst with a score better than grade 3BB was considered fair. Embryos that scored greater than grade $3 \mathrm{CC}$ on day 4 to 7 were vitrified.

To preserve the value of the concept, a robust cryopreservation program is mandatory. The vitrification and warming procedures were performed according to the Cryotop Safety Kit manual (Kitazato Co, Japan) at room temperature. In the vitrification procedures, blastocysts were equilibrated for 12-15 min in an equilibration solution and then washed and floated for 45-60 s in a vitrification solution (VS). Subsequently, blastocysts were placed on the Cryotop sheet with a minimum volume of VS. Immediately, the Cryotop sheets were submerged into liquid nitrogen. In the warming procedures, the Cryotop sheets were placed into a thawing solution and incubated at $37^{\circ} \mathrm{C}$ within $1 \mathrm{~s}$. After $1 \mathrm{~min}$, the blastocysts were placed in a diluent solution for 3 min. Finally, the blastocysts were placed in a washing solution for $5 \mathrm{~min}$. The blastocysts were cultured at $37^{\circ} \mathrm{C}$ for at least $3 \mathrm{~h}$ before ET.

\section{Endometrial preparation and frozen embryo transfer}

Thawed embryo transfers were performed in hormone replacement cycles. Endometrial preparation was undertaken by incremental doses of transdermal estradiol (ESTRANA® Tapes 0.72 mg; Hisamitsu Pharmaceutical Co., Inc., Tokyo, Japan). After confirming that an endometrial thickness greater than 8 mm by ultrasonography, oral progestin (chlormadinone acetate; Lutoral ${ }^{\circledR}$, Fuji Pharm, Tokyo, Japan) and transvaginal natural progesterone (LUTINUS ${ }_{8}$, Ferring Pharma; or OneCrinone ${ }^{\circledR}$, Merck Biopharm) were administered daily. Blastocyst transfer was performed on day 5 or 6 of progesterone administration.

\section{Pregnancy diagnosis}

Clinical pregnancy was defined as a pregnancy when a gestational sac was observed in the uterus by ultrasound. Ongoing pregnancy was defined as a pregnancy in which fetal cardiac activity was detected by ultrasound beyond 9 weeks.

\section{Statistical analysis}

Chi-square test and $t$ tests were performed to compare the differences between the two groups. A statistically significant difference was defined as $P<0.05$. 


\section{Results}

A total of 101 SF courses were initiated in 88 patients, and 485 OR cycles were performed between June 2017 and October 2018 . Patient characteristics were as follows: average age at the start of treatment, $39.5 \pm 3.8$ years (range, $27-46$ years); average number of previous IVF attempts, $3.6 \pm 4.2$ (range, $0-23$ ); and average AMH level, $0.81 \pm 0.71 \mathrm{ng} / \mathrm{mL}$ (range, $0.03-5.62 \mathrm{ng} / \mathrm{mL}$ ), which was obtained at the patient's first outpatient examination, on average 1.1 years before the start of the SF course. All the course attendants could not achieve ongoing pregnancy in the preceding conventional treatment at our clinic or at other institutions, including very severe patients with repeated unsuccessful treatments who were close to considering treatment termination. Data are presented as mean $\pm S D$.

An average $4.8 \pm 1.0$ (range, 1-8; median, 5) cycles of OR were performed during the 90-day treatment period, resulting in an average of $12.6 \pm 6.2$ (range, $1-$ 34 , median, 11) total harvested oocytes, $8.1 \pm 4.5$ (range, 1-23; median, 6) 2PN embryos, $4.8 \pm 3.4$ (range, 0-17; median, 4) vitrified embryos, and $2.7 \pm 2.8$ (range, $0-12$, median, 2) fair-grade blastocysts (Table 1).

Table 1

Clinical outcomes of the 3-month treatment course

\begin{tabular}{|c|c|c|c|}
\hline Results per treatment course $(n=101)$ & Average (mean $\pm \mathrm{SD})$ & $\begin{array}{l}\text { Range } \\
\text { (min-n }\end{array}$ & Median \\
\hline Attempted number of oocyte retrievals & $4.8 \pm 1.0$ & $1-8$ & 5 \\
\hline Number of retrieved oocytes & $12.6 \pm 6.2$ & $1-34$ & 11 \\
\hline Number of 2PN embryos & $8.1 \pm 4.5$ & $1-23$ & 6 \\
\hline Number of vitrified embryos & $4.8 \pm 3.4$ & $0-17$ & 4 \\
\hline Number of fair-grade blastocysts (Gardner score > 3BB) & $2.7 \pm 2.8$ & $0-12$ & 2 \\
\hline
\end{tabular}

To examine the efficacy of more than three continuous trials, 485 OR cycles were divided into two groups. The first group included the first or second retrievals of the SF course (201 cycles, 529 oocytes), and the second group included attempts after the patient's third retrieval (284 cycles, 740 oocytes). The ratio of matured oocytes, 2PN embryos, available embryos, fair-grade blastocysts per total oocytes harvested, number of available embryos, and fair blastocysts per OR cycle were reviewed and compared. In the first and second attempts, the maturation rate was $81.1 \%(429 / 529)$, the $2 \mathrm{PN}$ rate was $64.1 \%(339 / 529)$, the rate of available embryos was $35.9 \%$ (190/529), and the rate of fair-grade embryos was $19.8 \%(105 / 529)$. The number of available embryos per OR cycle was 0.95 $(191 / 201)$, and the number of fair-grade blastocysts was $0.52(105 / 201)$. On the other hand, after the third OR attempt, these parameters were as follows: maturation rate $81.6 \%(604 / 740), 2 \mathrm{PN}$ rate $64.7 \%$ (479/740), rate of available embryos $39.3 \%(291 / 740)$, and rate of fair-grade embryos $22.7 \%$ (168/740). The number of available embryos per OR cycle was 1.02 (289/284), and the number of fair-grade blastocysts was 0.59 (168/284). There were no statistically significant differences in any parameter between the two groups, although latter trials in the SF course tend to result in an increased rate of viable embryos and fair blastocysts (Table 2).

Table 2

Comparison of embryo parameters between the initial two attempts and the latter attempts

\begin{tabular}{|c|c|c|c|}
\hline & $\begin{array}{l}\text { OR at first and second trial (201 } \\
\text { cycles) }\end{array}$ & $\begin{array}{l}\text { OR at third and latter trial ( } 284 \\
\text { cycles) }\end{array}$ & $\begin{array}{l}P \\
\text { value }\end{array}$ \\
\hline $\begin{array}{l}\text { Total number of oocytes and number per OR (average; min- } \\
\text { max) }\end{array}$ & $529(2.63 ; 0-11)$ & $740(2.61 ; 0-12)$ & - \\
\hline $\begin{array}{l}\text { Rate of matured oocytes } \\
\text { (No. of Mll oocyte/total No. of oocytes) }\end{array}$ & $81.1 \%(429 / 529)$ & $81.6 \%(604 / 740)$ & n.s. \\
\hline $\begin{array}{l}\text { Rate of 2PN embryos } \\
\text { (No. of 2PN embryos/total No. of oocytes) }\end{array}$ & $64.1 \%(339 / 529)$ & $64.7 \%(479 / 740)$ & n.s. \\
\hline $\begin{array}{l}\text { Rate of available embryos } \\
\text { (No. of vitrified embryos/total No. of oocytes) }\end{array}$ & $35.9 \%(190 / 529)$ & $39.3 \%(291 / 740)$ & n.s. \\
\hline $\begin{array}{l}\text { Rate of fair-grade blastocysts } \\
\text { (No. of fair-grade embryos/total No. of oocytes) }\end{array}$ & $19.8 \%(105 / 529)$ & $22.7 \%(168 / 740)$ & n.s. \\
\hline No. of available embryos per OR & $0.95(191 / 201)$ & $1.02(289 / 284)$ & n.s. \\
\hline No. of fair-grade blastocysts per OR & $0.52(105 / 201)$ & $0.59(168 / 284)$ & n.s. \\
\hline OR, oocyte retrieval; n.s, not significant & & & \\
\hline
\end{tabular}


We next examined the efficacy of luteal-phase ORs. For this analysis, the group was divided into two groups: the FPS and LPS groups. FPS includes the cycles in which ovarian stimulation began in the early follicular phase (343 cycles, 973 oocytes), and LPS includes cycles in which ovarian stimulation began in the luteal phase (142 cycles, 296 oocytes). The ratio of matured oocytes in both groups, FPS and LPS, was $80.7 \%$ (785/973) and 83.8\% (248/296), respectively. The 2 PN rate was $63.1 \%(614 / 973)$ and $68.9 \%$ (204/296), the rate of available embryos was $36.5 \%(355 / 973)$ and $42.6 \%(126 / 296)$, and the rate of fair-grade blastocysts was $19.6 \%(191 / 973)$ and $27.7 \%$ (82/296), respectively. The number of available embryos per OR cycle was 1.03 (353/343) and $0.89(126 / 142)$, and the number of fair-grade blastocysts was $0.56(192 / 343)$ and $0.58(82 / 142)$, respectively. The rate of fair-grade blastocysts was significantly higher in the LPS group than in the FPS group $(p<0.01)$. Although the number of retrieved oocytes in the LPS group was smaller than in the FPS group, the number of fairgrade blastocysts was not reduced. Thus, treatment efficacy in LPS is not diminished, and OR in the LPS is worth attempting for patients with DOR (Table 3 ).

Table 3

Comparison of embryo parameters between FPS and LPS cycles

\begin{tabular}{|c|c|c|c|}
\hline & FPS (343 cycles) & LPS (142 cycles) & $P$ value \\
\hline Total number of oocytes and number per OR (average; min-max) & $973(2.84 ; 0-12)$ & $296(2.08 ; 0-11)$ & - \\
\hline $\begin{array}{l}\text { Rate of matured oocytes } \\
\text { (No. of Mll oocytes/total no. of oocytes) }\end{array}$ & $80.7 \%(785 / 973)$ & $83.8 \%(248 / 296)$ & n.s. \\
\hline $\begin{array}{l}\text { Rate of } 2 \mathrm{PN} \text { embryos } \\
\text { (No. of } 2 \mathrm{PN} \text { embryos/total no. of oocytes) }\end{array}$ & $63.1 \%(614 / 973)$ & $68.9 \%(204 / 296)$ & n.s. \\
\hline $\begin{array}{l}\text { Rate of available embryos } \\
\text { (No. of vitrified embryos/total no. of oocytes) }\end{array}$ & $36.5 \%(355 / 973)$ & $42.6 \%(126 / 296)$ & n.s. \\
\hline $\begin{array}{l}\text { Rate of fair-grade blastocyst } \\
\text { (No. of fair-grade embryo/total no. of oocytes) }\end{array}$ & $19.6 \%(191 / 973)$ & $27.7 \%(82 / 296)$ & $P<0.01$ \\
\hline No. of available embryos per OR & $1.03(355 / 343)$ & $0.89(126 / 142)$ & n.s. \\
\hline No. of fair-grade blastocysts per OR & $0.56(191 / 343)$ & $0.58(82 / 142)$ & n.s. \\
\hline FPS, follicular-phase stimulation; LPS, luteal-phase stimulation; PN & 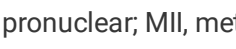 & aphase II; OR, oocyt & retrieval \\
\hline
\end{tabular}

Next, we present an impressive clinical course in which the patient underwent eight OR trials over 90 days, which was the highest number of trials in this study. At the beginning of the course, she was 40 years old with DOR and an AMH value of $0.79 \mathrm{ng} / \mathrm{mL}$ (which was checked 4 months before the course started). Ten oocytes, including nine MII oocytes, were collected during the course, and six blastocysts were vitrified following fertilization and embryo culture. She experienced four consecutive retrievals following LPS, the corpus luteum continued to exist, and she had no menstruation during the period. We did not attempt a fifth consecutive LPS because of her personal reasons and re-started stimulation for 6 th OR after the next menstruation. Follicle maturation was observed at approximate 10-day intervals, resulting in eight OR trials. She became pregnant after her first embryo transfer with the embryo derived from the fourth consecutive and luteal-phase retrieval, and she delivered a healthy baby (Table 4). 
Table 4

Clinical course of the women who experienced eight trials of OR during the SF course

\begin{tabular}{|c|c|c|c|c|c|c|c|}
\hline & OR1 & OR2 & OR3 & OR4 & OR5 & OR6 & OR7 \\
\hline $\begin{array}{l}\text { Stimulation } \\
\text { start at FP } \\
\text { or LP }\end{array}$ & $\mathrm{FP}$ & LP & LP & LP & LP & FP & LP \\
\hline $\begin{array}{l}\text { Stimulation } \\
\text { start on }\end{array}$ & $\mathrm{d} 4$ & (mid-luteal) & Next day of OR & Next day of OR & Next day of OR & d1 & Next day of OR \\
\hline $\begin{array}{l}\text { Ovarian } \\
\text { stimulation }\end{array}$ & $\begin{array}{l}\mathrm{CC}+\mathrm{hMG}^{* 6} 6 \\
\text { days }\end{array}$ & hMG*6 daily & $\begin{array}{l}\mathrm{CC}+ \\
\mathrm{hMG} * 2 \text { days }\end{array}$ & $\begin{array}{l}\mathrm{CC}+ \\
\mathrm{hMG} * 3 \text { days }\end{array}$ & $\mathrm{CC}+\mathrm{hMG} * 3$ days & $\begin{array}{l}\mathrm{CC}+\mathrm{hMG}^{* 6} 6 \\
\text { days }\end{array}$ & hMG *6 daily \\
\hline $\begin{array}{l}\text { Hormone } \\
\text { profile on } \\
\text { the day of } \\
\text { trigger } \\
\text { (E2/P4/LH) } \\
\text { (* day } \\
\text { before } \\
\text { trigger) }\end{array}$ & $576.4 / 0.16 / 11.7$ & $447.2 / 0.19 / 2.57$ & $900.7 / 6.42 / 7.13$ & $1230 / 0.46 / 5.79$ & $\begin{array}{l}\text { 1667/19.56/4.88 } \\
\text { ( } 2 \text { days before) }\end{array}$ & $\begin{array}{l}683.9 / 0.05 / 5.83 \\
\text { (1 day before) }\end{array}$ & $1372.0 / 23.88 / 11.06$ \\
\hline $\begin{array}{l}\text { Day of } \\
\text { menses } \\
\text { cycle on OR }\end{array}$ & Day 20 & Day 4 & Day 13 & Day 24 & Day 35 & Day 14 & Day 22 \\
\hline $\begin{array}{l}\text { OR-OR } \\
\text { interval(day) }\end{array}$ & 0 & 17 & 9 & 11 & 11 & 23 & 8 \\
\hline $\begin{array}{l}\text { Number of } \\
\text { retrieved } \\
\text { oocytes }\end{array}$ & 1 & 1 & 1 & 2 & 1 & 1 & 1 \\
\hline $\begin{array}{l}\text { Number of } \\
\text { vitrified } \\
\text { embryos }\end{array}$ & 1 & 0 & 1 & 1 & 1 & 1 & 1 \\
\hline $\begin{array}{l}\text { Embryo } \\
\text { status, } \\
\text { Gardner's } \\
\text { score }\end{array}$ & D5 4BC & $\begin{array}{l}\text { Attested at } \\
\text { morula }\end{array}$ & D6 4BB & D4 5AB & D6 4BC & D5 4CB & D5 5AA \\
\hline
\end{tabular}

OR, oocyte retrieval; LP, luteal phase; FP, follicular phase; LH, luteinizing hormone; CC, clomiphene citrate; hMG, human menopausal gonadotropin

Impressive clinical course with eight OR trials over 90 days, the highest number of trials in this study.

At the time of writing (March 2021), the clinical outcomes of the SF course after subsequent embryo transfers were reviewed. Thirteen patients have tried the course twice, and 88 women have received thawed embryo transfers. Transferred embryos were selected based on morphology, irrespective of timing of ORs and FPS or LPS. Sixty-two women have achieved clinical pregnancy, and 49 women have achieved an ongoing pregnancy. Forty-seven women have delivered healthy babies. Twenty-six of these babies were derived from the oocyte in the latter ORs of the course, and thirteen were derived from LPS cycles. In addition, eight women have conceived second babies derived from the oocytes collected during the course. Eighteen women have terminated fertility treatment without ongoing pregnancy. There are 15 women who are still undergoing treatment and have not achieved an ongoing pregnancy, but these women still have reserved embryos (Fig. 1).

\section{Discussion}

To the best of our knowledge, this is the first report of the clinical trial, in which more than three consecutive ORs are used to improve the chance of childbearing for the patients who have severe difficulty achieving pregnancy.

In this study, we extended the concept of random-start and DuoStim and furthermore intended to acquire an increasing number of oocytes to maximize the chance of obtaining a potential embryo. It is estimated that, in order to obtain at least one euploid embryo, the collection of $5,7,10$, and 20 oocytes is required in women aged $35-37,38-40,41-42$, and $>42$ years, respectively [21]. In our clinical setting, which includes many patients who are refractory to treatment, collecting enough oocytes to achieve a pregnancy in these patients within two or three trials of OR is a difficult task. In Japan, many women with advanced maternal age or DOR have been undergoing ORs and embryo transfers for many years. They are suffering from both physical and mental stress and feel as though they are in a dark tunnel with no visible exit. This treatment environment is particularly severe in Japan for several reasons, including that oocyte donation is not permitted, preimplantation genetic testing for aneuploidy (PGT-A) is regulated and not available in our clinic, and single-embryo transfer is strongly recommended. Success in this restricted situation is a most challenging task for clinicians. Therapeutic efficacy is low, no matter what efforts we undertake. When patients experience miscarriage, months of time has been lost until the next trial. This is a heavy blow to all women, and the resulting physical and psychological damages can be endless. Thus, the SF technique can help save precious time and decrease the time lost after miscarriage.

Another problem arising from this treatment might be an increased physical burden, mental stress, and economic load. Although it depends on the clinic's policy, this course may be more cost effective. We set a low fixed treatment cost for 3 months so that most patients could repeat the OR without worrying about the cost. Looking back over the SF course, 3 months of treatment might be appropriate. Because one of the stressors patients face is an uncertain future, setting one periodic goal helps them to retain positive feelings and relieve the stress. Treatment discontinuation in poor responders is a crucial issue, 
but none of the patients dropped out during the period owing to a failed attempt. The accessibility of patients is one of the main advantages of this limited time-frame consecutive strategy. Some patients could not obtain a successful result and thus terminated their infertility treatment after the SF course and subsequent transfer. They did not have feelings of regret because they believed they did everything they could. On the other hand, 13 women could not end their treatment and hoped to start a second trial of the SF course. An additional problem was our institution's limited capacity. Increasing the number of ORs obviously led to an increased workload of doctors, nurses, embryologists, and other staff members. Seven or eight OR trials over 3 months obtained good outcomes that went beyond our expectations. The flat-rate and patient-friendly system may be challenging for the management in terms of balancing patients, staff, and clinic; however, the positive aspect is that good results were achieved and many couples were satisfied.

Three follicle recruitment theories have been postulated to date. One is the classical "single recruitment" theory, and the others include the "wave" theory and "continuous recruitment" theory [22]. In humans, the single recruitment theory has traditionally supported that only a single major wave of follicle development occurs during the intraovulatory period. However, previous studies have demonstrated the appearance of more than one wave of follicular growth within a cycle, suggesting the presence of obtainable follicles during the luteal phase [23]. In fact, some recent studies using random ovarian stimulation for women with cancer [24-26] or luteal-phase ovarian stimulation for poor responders (DuoStim) demonstrated that LPS is an adequate method for obtaining a sufficient number of competent oocytes [27]. In addition, we experienced a thought-provoking case of folliculogenesis. This patient underwent eight ORs over 90 days, including four consecutive retrievals after LPS, during which no menstruation occurred. In this course, we commonly experienced two to three serial luteal-phase ORs in many other patients. It is thought that follicle maturation could occur at approximate 10-day intervals and that consecutive luteal-phase follicle maturation could possibly be endless. Although the biological process for folliculogenesis is not yet completely understood, this intriguing evidence add some clues in favor of the "continuous recruitment" theory, in that recruitable antral follicles are continuously present in the ovaries [20].

In the SF course, an average of 4.9 trials of ORs were performed on average (range, 1-8) over the 90-day period among 101 women with severe conditions. This translates to an OR chance 1.6 times higher than a conventional monthly trial, resulting in more oocytes (average $12.6 \pm 6.2$ oocytes) over the duration of the course. Similar to previous reports on DuoStim and LPS [28], we found no significant difference between FPS and LPS in all parameters, but we noted a superior rate of fair-grade blastocysts per retrieved oocyte in the LPS group. In addition, three or more, or even eight, consecutive trials did not diminish treatment efficacy or embryo competence. All of these results verify that ovarian stimulation can begin at any phase of the menstrual cycle and can be repeated any number of times, irrespective of menstrual cycle. Thus, the SF course can be used to maximize the potency of refractory patients by obtaining more chances for $\mathrm{OR}$, accumulating the greatest number of oocytes, and acquiring more competent embryos in a limited period. Although this was a retrospective clinical study in a heterogenous patient population, the delivery of healthy babies, and also siblings, proves the strategy's clinical utility.

Without new technology to restore intrinsic embryo competence, clinicians can only improve on the currently available methods, among which are the selection of proper ovarian stimulation and time management. One proposed strategy in patients with poor response is embryo accumulation and vitrification [29-31] with double stimulation in the follicular and luteal phase of the same ovarian cycle (i.e., DuoStim) [32-35]. This method offers the possibility of the maximum attempts at oocyte collection to obtain the highest number of eggs in a single menstrual cycle. Our strategy, the "SF," further extends the treatment period from a single menstrual cycle to 3 months, without regard for the menstrual cycle. Increasing the chance of OR just a bit more and collecting even a few more competent embryos in a limited period has contributed to the increased cumulative live birth rate and reduced the time to achieve pregnancy. The availability of PGT-A in Japan would also contribute to reducing the treatment period by avoiding futile embryo transfer or miscarriage [36]. The treatment period could be reconsidered in each patient according to the number of euploid embryos acquired or their background, such as maternal age and family planning. A result of no euploid embryos provides patients the next chance of OR at in earlier stage of their lives or allows them to decide on treatment termination and pursue a new stage in their life. This course might hold not only medical, but also social, meaning. The most suitable target population for the SF course might include very severe patients with DOR, with shortened menstrual cycles, and a low level of luteal-phase hormone, in which luteal-phase oocyte maturation could occur easily and early because of constantly high concentrations of FSH [37]. Most of these patients could experience more ORs (e.g., five to eight) than average, but some of these patients with further severe DOR, who are perimenopausal, or who rarely ovulate during the period could achieve only one or two OR trials; thus, the treatment would not be effective, and they were not retrospectively eligible. On the other hand, some of these patients might decide to stop treatment after two or three ORs (i.e., before the duration of the course is over), because they have acquired a satisfactory number of embryos. Further examination is required to find the most suitable target for this treatment and the most suitable period for each patient with the aim of maximizing this strategy's utility.

\section{Conclusion}

This study demonstrated that embryos derived from LPS or from consecutive OR over a 3-month duration have similar development potential as conventional stimulation. SF, which is a strategy of periodic consecutive ORs, has been proven to enhance the possibility of childbearing by increasing the chance of OR and increasing the number of oocytes and competent embryos within the limited time. Our experience provides new insight into folliculogenesis, in which oocyte maturation could occur every 10 days, and continuous luteal-phase follicle maturation could occur repeatedly, during a period in which no menstruation occurs. This new concept represents an intriguing protocol and provides a new and effective choice for infertility treatment, especially for timesensitive women with advanced maternal age or DOR. This trial certainly demands further investigation to define the proper target and period and to assess the safety and actual clinical efficiency. Managing the SF course requires maintaining an overall balance between not only medical matters but also psychologic and economic issues.

\section{Abbreviations}

FPS: follicular-phase stimulation

LPS: luteal-phase stimulation 
OR: oocyte retrieval

DOR: decreased ovarian reserve

SF: squeeze and freeze

\section{Declarations}

Ethics approval: The study was approved by the clinic's Institutional Review Board and was carried out in accordance with the Code of Ethics of the World Medical Association (Declaration of Helsinki) for experiments involving humans.

Consent to participate: All participants provided informed consents after receiving counseling for this infertility treatment.

Consent for publication: not applicable

Availability of supporting data: The data supporting the findings of this study are available within the article and its supplementary materials and from the corresponding author on request.

Competing interests: The authors declare that they have no competing interests.

Funding: This research did not receive any specific grant from funding agencies in the public, commercial, or not-for-profit sectors.

Authors' contributions: YM designed and executed the study, drafted the manuscript and contributed to critical discussion. FO and MS performed the data analysis. NS, AS, and TM were involved in study execution.

Acknowledgements: The authors would like to thank Enago (www.enago.jp) for the English language review.

\section{References}

1. Liu K, Case A, Reproductive Endocrinology and Infertility Committee.Advanced reproductive age and fertility. J Obstet Gynaecol Can. 2011;33:1165-75.

2. Sauer MV. Reproduction at an advanced maternal age and maternal health. Fertil Steril. 2015;103:1136-

3. Ubaldi FM, Cimadomo D, Vaiarelli A, Fabozzi G, Venturella R, Maggiulli R, Mazzilli R, Ferrero S, Palagiano A, Rienzi L. Advanced maternal age in IVF: Still a challenge? The present and the future of its treatment. Front Endocrinol. (Lausanne) 2019;10:94.

4. Cimadomo D, Fabozzi G, Vaiarelli A, Ubaldi N, Ubaldi FM, Rienzi L.Impact of maternal age on oocyte and embryo competence. Front Endocrinol. (Lausanne) 2018a;29:327.

5. Franasiak JM, Forman EJ, Hong KH, Werner MD, Upham KM, Treff NR, Scott Jr RT. The nature of aneuploidy with increasing age of the female partner: a review of 15,169 consecutive trophectoderm biopsies evaluated with comprehensive chromosomal screening. Fertil Steril. 2014;101:656-63.

6. Goldman MB, Thornton KL, Ryley D, Alper MM, Fung JL, Hornstein MD, Reindollar RH. A randomized clinical trial to determine optimal infertility treatment in older couples: the Forty and Over Treatment Trial (FORT-T). Fertil Steril. 2014;101:1574-81.

7. Wennberg AL, Opdahl S, Bergh C, Aaris Henningsen AK, Gissler M, Romundstad LB, Pinborg A, Tiitinen A, Skjærven R, Wennerholm UB. Effect of maternal age on maternal and neonatal outcomes after assisted reproductive technology. Fertil Steril. 2016;106:1142-9.

8. Kuwayama M, Vajta G, Kato O, Leibo SP.Highly efficient vitrification method for cryopreservation of human oocytes. Reprod Biomed Online. 2005;11:3008.

9. Toner JP, Coddington CC, Doody K, Van Voorhis B, Seifer DB, Ball GD, Luke B, Wantman E. Society for assisted reproductive technology and assisted reproductive technology in the United States: a 2016 update. Fertil Steril. 2016;106:541-6.

10. Mizrachi Y, Horowitz E, Farhi J, Raziel A, Weissman A.Ovarian stimulation for freeze-all IVF cycles: a systematic review. Hum Reprod Update. 2020;26:118-35.

11. Cakmak H, Kat Z, Cedars MI, Rosen MP. Effective method for emergency fertility preservation: random-start controlled ovarian stimulation. Fertil Steril.2013;100:1673-80.

12. Rashidi BH, Tehrani ES, Ghaffari F. Ovarian stimulation for emergency fertility preservation in cancer patients: a case series study. Gynecol Oncol Rep.2014;10:19-21.

13. Labarta E. A new strategy proposed for women with poor ovarian response. Fertil Steril. 2020;113:76-7.

14. Vaiarelli A, Cimadomo D, Petriglia C, Conforti A, Alviggi C, Ubaldi N, Ledda S, Ferrero S, Rienzi L, Ubaldi FM. DuoStim-a reproducible strategy to obtain more oocytes and competent embryos in a short time-frame aimed at fertility preservation and IVF purposes. A systematic review. Ups J Med Sci. 2020;125:121-30.

15. Vaiarelli A, Cimadomo D, Conforti A, Schimberni M, Giuliani M, D’Alessandro P, Colamaria S, Alviggi C, Rienzi L, Ubaldi FM. Luteal phase after conventional stimulation in the same ovarian cycle might improve the management of poor responder patients fulfilling the Bologna criteria: a case series. Fertil Steril. 2020;113:121-30

16. Vaiarelli A, Cimadomo D, Alviggi E, Sansone A, Trabucco E, Dusi L, Buffo L, Barnocchi N, Fiorini F, Colamaria S, Giuliani M. The euploid blastocysts obtained after luteal phase stimulation show the same clinical, obstetric and perinatal outcomes as follicular phase stimulation-derived ones: a multicenter study. Hum Reprod 2020;35:2598-608. 
17. Li Y, Yang W, Chen X, Li L, Zhang Q, Yang D. Comparison between follicular stimulation and luteal stimulation protocols with clomiphene and HMG in women with poor ovarian response. Gynecol Endocrinol. 2016;32:74-7.

18. Qin N, Chen Q, Hong Q, Cai R, Gao H, Wang Y, Sun L, Zhang S, Guo H, Fu Y, Ai A. Flexibility in starting ovarian stimulation at different phases of the menstrual cycle for treatment of infertile women with the use of in vitro fertilization or intracytoplasmic sperm injection. Fertil Steril. 2016;106:334-41.

19. Wang N, Wang Y, Chen Q, Dong J, Tian H, Fu Y, Ai A, Lyu Q, Kuang Y. Luteal-phase ovarian stimulation versus conventional ovarian stimulation in patients with normal ovarian reserve treated for IVF: a large retrospective cohort study. Clin Endocrinol. 2016;84:720-8.

20. Ferraretti AP, La Marca A, Fauser BC, Tarlatzis B, Nargund G, Gianaroli L, ESHRE working group on Poor Ovarian Response Definition.ESHRE consensus on the definition of 'poor response' to ovarian stimulation for in vitro fertilization: the Bologna criteria. Hum Reprod. 2011;26:1616-24.

21. Vaiarelli A, Cimadomo D, Ubaldi N, Rienzi L, Ubaldi FM. What is new in the management of poor ovarian response in IVF?Curr Opin Obstet Gynecol. 2018b;30:155-62.

22. Baerwald AR, Adams GP, Pierson RA. Ovarian antral folliculogenesis during the human menstrual cycle: a review. Hum Reprod Update. $2012 ; 18: 73-91$.

23. Baerwald A, Adams G, Pierson R. Characteristics of ovarian follicular wave dynamics in women. Biol Reprod. 2003;69:1023-31.

24. Anderson RA, Kinniburgh D, Baird DT. Preliminary experience of the use of a gonadotrophin-releasing hormone antagonist in ovulation induction/in-vitro fertilization prior to cancer treatment. Hum Reprod. 1999;14:2665-8.

25. de Melo Gagliato D, Gonzalez-Angulo AM, Lei X, Theriault RL, Giordano SH, Valero V, Hortobagyi GN, Chavez-MacGregor M. Clinical impact of delaying initiation of adjuvant chemotherapy in patients with breast cancer. J Clin Oncol. 2014;32:735-44.

26. von Wolff M, Thaler CJ, Frambach T, Zeeb C, Lawrenz B, Popovici RM, Strowitzki T. Ovarian stimulation to cryopreserve fertilized oocytes in cancer patients can be started in the luteal phase. Fertil Steril. 2009;92:1360-5.

27. Kuang Y, Chen Q, Hong Q, Lyu Q, Ai A, Fu Y, Shoham Z. Double stimulations during the follicular and luteal phases of poor responders in IVF/ICSI programmes (Shanghai protocol). Reprod Biomed Online. 2014a;29:684-91.

28. Ubaldi FM, Capalbo A, Vaiarelli A, Cimadomo D, Colamaria S, Alviggi C, Trabucco E, Venturella R, Vajta G, Rienzi L. Follicular versus luteal phase ovarian stimulation during the same menstrual cycle (DuoStim) in a reduced ovarian reserve population results in a similar euploid blastocyst formation rate: new insight in ovarian reserve exploitation. Fertil Steril. 2016;105:1488-95.

29. Chatziparasidou A, Nijs M, Moisidou M, Chara O, loakeimidou C, Pappas C, Christoforidis N. Accumulation of oocytes and/or embryos by vitrification, a new strategy for managing poor responder patients undergoing pre implantation diagnosis. F1000 Res. 2013;2:240.

30. Cobo A, Garrido N, Crespo J, Jose R, Pellicer A. Accumulation of oocytes: a new strategy for managing low-responder patients. Reprod Biomed Online. 2012;24:424-32.

31. Martinez F, Barbed C, Parriego M, Sole M, Rodriguez I, Coroleu B. Usefulness of oocyte accumulation in low ovarian response for PGS. Gynecol Endocrinol. 2016;32:577-80.

32. Cimadomo D, Vaiarelli A, Colamaria S, Trabucco E, Alviggi C, Venturella R, Alviggi E, Carmelo R, Rienzi L, Ubaldi FM. Luteal phase anovulatory follicles result in the production of competent oocytes: intra-patient paired case-control study comparing follicular versus luteal phase stimulations in the same ovarian cycle. Hum Reprod. 2018b;33:1442-8.

33. Kuang Y, Hong Q, Chen Q, Lyu Q, Ai A, Fu Y, Shoham Z. Luteal-phase ovarian stimulation is feasible for producing competent oocytes in women undergoing in vitro fertilization/intracytoplasmic sperm injection treatment, with optimal pregnancy outcomes in frozen-thawed embryo transfer cycles. Fertil Steril. 2014b;101:105-11.

34. Vaiarelli A, Venturella R, Vizziello D, Bulletti F, Ubaldi FM. Dual ovarian stimulation and random start in assisted reproductive technologies: from ovarian biology to clinical application. Curr Opin Obstet Gynecol. 2017;29:153-9.

35. Vaiarelli A, Cimadomo D, Trabucco E, Vallefuoco R, Buffo L, Dusi L, Fiorini F, Barnocchi N, Bulletti FM, Rienzi L, Ubaldi FM. Double stimulation in the same ovarian cycle (DuoStim) to maximize the number of oocytes retrieved from poor prognosis patients: a multicenter experience and SWOT analysis. Front Endocrinol. 2018a;9:317.

36. Sato T, Sugiura-Ogasawara M, Ozawa F, Yamamoto T, Kato T, Kurahashi H, Kuroda T, Aoyama N, Kato K, Kobayashi R, Fukuda A, Utsunomiya T, Kuwahara A, Saito H, Takeshita T, Irahara M. Preimplantation genetic testing for aneuploidy: a comparison of live birth rates in patients with recurrent pregnancy loss due to embryonic aneuploidy or recurrent implantation failure. Hum Reprod. 2019;34:2340-8.

37. Hale GE, Zhao X, Hughes CL, Burger HG, Robertson DM, Fraser IS. Endocrine features of menstrual cycles in middle and late reproductive age and the menopausal transition classified according to the staging of reproductive ageing workshop (STRAW) staging system $\mathrm{J}$ Clin Endocrinol Metab.

2007;92:3060-7.

\section{Figures}




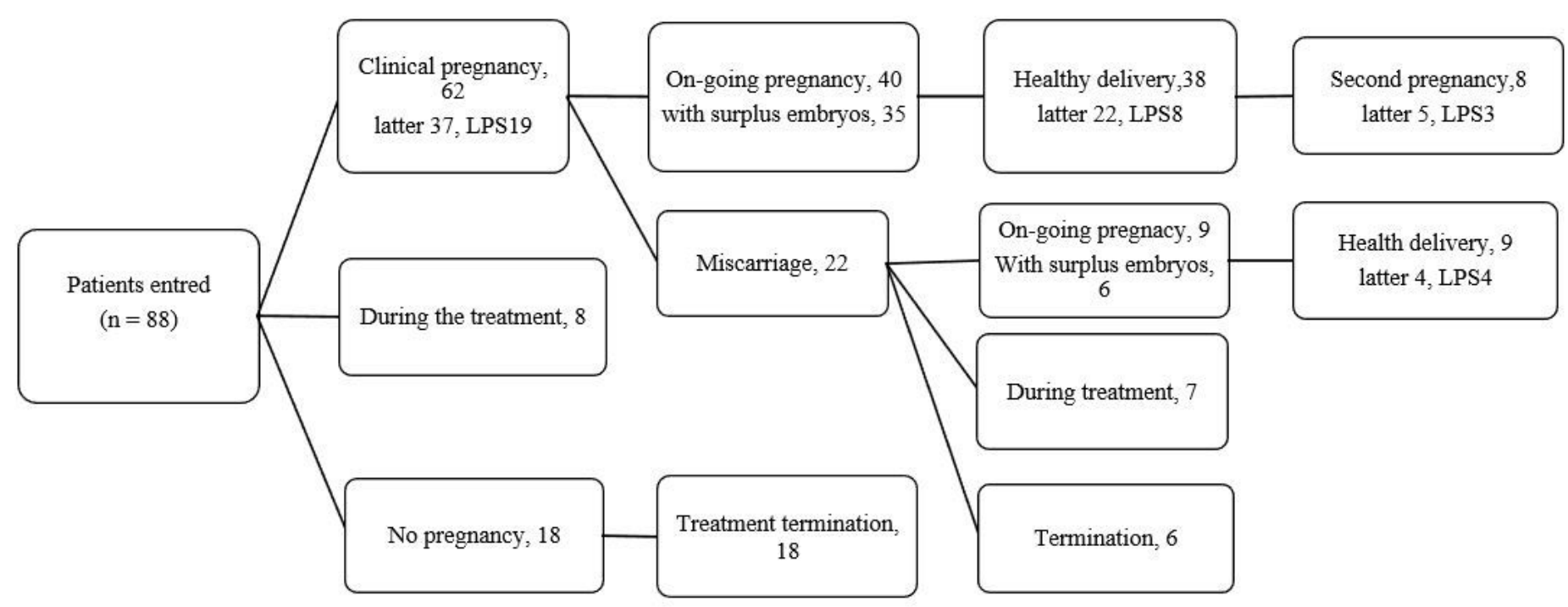

\section{Figure 1}

Clinical outcomes after the SF course (March 2021) Latter, derived from the embryos collected from the latter trial of the course (later than the third trial); LPS, derived from the embryos collected from the LPS (luteal-phase stimulation) 